\title{
Choosing the right survey: the lung cancer surgery
}

\author{
Cecilia Pompili ${ }^{1,2}$, Michael Koller ${ }^{3}$, Galina Velikova ${ }^{1}$ \\ ${ }^{1}$ Section of Patient Centred Outcomes Research, Leeds Institute for Medical Research at St. James's, University of Leeds, Leeds, UK; ${ }^{2}$ Division of \\ Thoracic Surgery, St. James's University Hospital, Leeds, UK; ${ }^{3}$ Center for Clinical Studies Regensburg, University of Regensburg, Regensburg, \\ Germany \\ Contributions: (I) Conception and design: All authors; (II) Administrative support: C Pompili; (III) Provision of study materials or patients: None; \\ (IV) Collection and assembly of data: All authors; (V) Data analysis and interpretation: All authors; (VI) Manuscript writing: All authors; (VII) Final \\ approval of manuscript: All authors. \\ Correspondence to: Dr. Cecilia Pompili. Section of Patient Centred Outcomes Research, Leeds Institute for Medical Research at St. James's, University \\ of Leeds, Leeds, UK; Division of Thoracic Surgery, St. James's University Hospital, Leeds, UK. Email: c.pompili@leeds.ac.uk.
}

\begin{abstract}
Radical and palliative treatments for non-small cell lung cancer (NSCLC) have faced a significant evolution during last decades, which in turn has modified their effects on the patient quality of life (QoL). QoL data collection is becoming methodologically rigorous, with published recommendations and societal statements. In lung cancer surgery, there is no standardization in collecting and analyzing this outcome yet. However, to face the evolution in lung cancer therapies, few initiatives like the European Organization for the Research and Treatment of Cancer (EORTC) lung cancer module update project or the PatientReported Outcomes Measurement Information System (PROMIS), have now the potential to help thoracic surgeons to collect QoL data with validated dynamic instruments to make this outcome comparable to the clinical ones. This review aims to give an overview of the available and most commonly used QoL questionnaires in lung cancer surgery in order to facilitate future research and practical implementation.
\end{abstract}

Keywords: Quality of life (QoL); patient-reported outcomes (PROs); thoracic surgery; patient centered care

Submitted Dec 10, 2019. Accepted for publication Dec 23, 2019.

doi: $10.21037 /$ jtd.2019.12.131

View this article at: http://dx.doi.org/10.21037/jtd.2019.12.131

\section{Introduction}

Advances in the diagnosis and treatments of non-small cell lung cancer have raised new important issues about patients' perspectives in the decision-making process.

For early-stage lung cancer for example, the introduction of stereotactic body radiotherapy (SABR) as the standard treatment for medically inoperable early-stage non-small cell lung cancer has introduced new challenge to patients considerate at high risk for surgery. Recent data suggest that in operable patients, SABR produces outcomes comparable to those of surgical resection (1). Understanding the evolution of quality of life (QoL) after surgical treatment for lung cancer by the surgeon may help the patient to participate proactively in the difficult decision-making process. In thoracic surgery in general, the use of QoL assessment has been certainly improved in the recent years (2), but its use in clinical practice remains underestimated. A recently published survey among European thoracic surgeons, showed a lack of standardized patient-reported outcomes (PROs) collection among this community with $88 \%$ of all surgeons currently not integrating these outcomes into their clinical practices (3). Marked discordance was also evident when asked about the type of instrument used to assess QoL. Among those collecting QoL information, a higher percentage of respondents (50\%) used the generic QoL questionnaire, while $48.5 \%$ of respondents introduced the administration of the cancer or lung cancer-specific tool.

Giving the lack of standardized practice in our specialty when collecting QoL and PROs information, this review aims to give an overview of the available and most commonly used QoL questionnaires in lung cancer surgery in order to facilitate future research and practical 
applicability.

\section{What and how to measure: QoL and PROs}

The World Health Organization (4) defined in 1995 QoL as "individuals' perceptions of their position in life in the context of their culture and value systems in which they live and in relation to their goals, expectations, standards and concerns".

Over the last decades however, the research area of QoL assessment has become more standardized and methodologically rigorous, with published recommendations and Societal statements. The most recent one from the 5th EORTC Quality of Life in Cancer Clinical Trials Conference updated definitions have been agreed by experts in oncological field including researchers, regulators, industry representatives, patients and patient advocates (5).

PROs is an umbrella term that embraces any outcome that is directly reported by the patient (in most cases via a standardized questionnaire). Health-related QoL is a multidimensional concept referring to the patient's subjective perception of the effect of their disease and treatment on physical, psychological and social aspects of daily life. PROs are becoming implemented in most of the clinical and surgical trials. It is increasingly recognized that the collection, interpretation and reporting of PROs in cancer clinical trials poses particular challenges (6).

For this reason, the Setting International Standards in Analyzing Patient-Reported Outcomes and Quality of Life Endpoints Data for Cancer Clinical Trials (SISAQOL) Consortium has been recently created to address this issue and to develop standards, guidelines, and recommendations for the analysis of PROs data in cancer trials (7-9).

The instruments currently used in lung cancer surgery are those which have been developed mostly to assess QoL in the oncological setting: these tools were focusing mainly on symptoms and functioning and their timeframes were linked to the weekly chemotherapy side-effect reports. No lung cancer surgery specific validated questionnaire has been developed and validated so far, diminishing the ability to draw definitive conclusions in our setting.

\section{Lung cancer surgery setting}

The ideal QoL questionnaire should be developed and validated according to strict guidelines verifying its reliability, validity, responsiveness to change and interpretability (10).
Authors have indicated that the selection of the QoL questionnaire should be performed among already validated tools (11). Questionnaire's characteristics to be considered are mostly related to the aim of the study, study population, measurement properties, study design issues, scoring and data analysis. Only a direct comparison within the same study/population may provide better justification for the selection of instrument. However, our group already demonstrated the issue of patient's burden in completing long questionnaires with similar questions (12) as attempted in other oncology specialties $(13,14)$. Recall bias cannot be completely ruled out and appear inherent to studies comparing two similar questionnaires.

Unfortunately, in our speciality the best timing to evaluate QoL has not been identified in previous published papers. The preoperative values seem to have a crucial role in this type of research. A reasonably long-term followup (more than 1 year) after surgical intervention may be desirable, since the patient may be biased during the first months in his judgment by factors such as the postoperative hospital stay, especially if complications occurred or the possibility of being treated with additional systemic therapies. Indeed, a shorter follow-up in QoL assessment may increase the response rate reducing the drops-out in questionnaires returns over-time.

Quite generally, we can distinguish between two types of questionnaires: generic and cancer specific. We also introduce recent projects which have the potential to change the QoL collection in lung cancer trials: the EORTC lung cancer module update, the EORTC Item Bank and the PROMIS.

\section{Generic questionnaires}

\section{SF-36}

The Medical Outcomes Study (MOS) Short Form 36item Health Survey (SF-36) has been developed in USA in 1997 for application in a wide range of medical conditions and to provide also valid normative data from the general population in different countries (15). One of its main features is that it can be adopted to compare health status between populations and between diseases. The 36 items contribute to assess health across eight domains: bodily pain (BP), general health perceptions (GH), mental health $(\mathrm{MH})$, physical functioning (PF), role limitations due to emotional health problems (RE), role limitations due to physical health problems (RP), social functioning (SF), and vitality (VT). Scores standardized to norms and weighted 
averages are used to create summary physical component summary (PCS) and mental component summary (MCS) scores on a standard scale. All scales contribute in different proportions to the scoring of both PCS and MCS measures. In the SF36v2, all health dimension scores are standardized to norms by using a linear transformation of data originally scored on a $0-100$ scale. Norm-based scores have a mean of 50 and a standard deviation of 10 . Any score $<50$ indicates a QoL below the general population mean. This feature helps the researchers to make a direct comparison of measures but most importantly to evaluate the long-term effectiveness of the treatment when the baseline assessment is not available. Several studies in thoracic surgery have used the SF-36v2 especially for its characteristic to allow a direct comparison with general populations (16-20).

\section{Health-economic evaluation questionnaire}

\section{Euroqol-5 Dimension (EQ-5D) questionnaire}

The EQ-5D is a generic measure widely used in healtheconomic evaluation (21). It has been developed by the EuroQol Group to describe and value health across a wide range of diseases $(22,23)$. It describes five single item dimensions: mobility, self-care, usual function status, pain and/or discomfort, and anxiety and/or depression. It is structured in only five questions and a visual analogue scale (VAS) evaluating the overall health status. This survey has been developed for self-completion by respondents and available in both paper and digital versions, but proxy versions are also available for populations in which selfcompletion is not possible. It is a very short questionnaire which takes, according to developers, only 5 minutes to be completed. The original questionnaire, asked respondents to choose between three options/levels; level 1, representing no problems; level 2, reflecting small or moderate problems; and level 3, indicating extreme problems (or "unable to"). A more recent version expanded the response choices from three to five levels (EQ-5D-5L) to face the frequently reported EQ-5D's ceiling effect (24).

The EQ-5D results can be presented as description of the single five dimensions or results of the EQ VAS as a measure of overall self-rated health status. Furthermore, it generates a single summary index value for health status. The EQ-5D summary index (index value) is derived by applying a formula that essentially attaches weights to each of the levels in each dimension, that can be compared according to the preferences of the general population of a country.
Only recently, and mostly for cost-utility analysis, this questionnaire has been used in surgical lung cancer patients $(25,26)$.

\section{Cancer and lung cancer specific questionnaires}

\section{European Organization for Research and Treatment of Cancer Quality of Life Questionnaire-Core Module (EORTC QLQ-C30)}

The EORTC QLQ-C30 is a 30-item cancer-specific instrument (27). Multi-trait scaling was used to determine five functional domain scales (physical, role, emotional, social and cognitive); additionally, one scale captures global QoL. Furthermore, the instrument also contains nine symptom scales capturing fatigue, nausea and vomiting, pain, dyspnoea, insomnia, appetite loss, constipation, diarrhoea and financial difficulties. The two scales for global health employ 7-point Likert scales; the other 28 items use 4-point Likert scales ranging from "not at all" to "very much". Each domain scale is transformed to a scale of $0-100$. For the functional and global scales higher scores indicate a better level of functioning; for the symptom scales, higher scores represent more severe symptoms. This instrument has been extensively tested for reliability and validity $(28,29)$. This questionnaire has been extensively used in our speciality (30-34). More recently a Summary Score $(\mathrm{SumSc})$ has been developed from this questionnaire and validated in lung cancer surgical patient $(35,36)$. The SumSc can avoid problems with potential type I errors that arise because of multiple testing when making comparisons based on the 15 outcomes generated by this whole survey. In addition, the use of the QLQ-C30 SumSc can reduce sample size requirements in clinical trials were QoL is a primary endpoint.

\section{European Organization for Research and Treatment of Cancer Quality of Life Questionnaire (EORTC QLQ- LC13)}

The EORTC QLQ-C30 core questionnaire (30 items) can be supplemented by the lung cancer module QLQ-LC13 (37). The QLQ-LC13 module evaluates with 13 questions, typical symptoms of lung cancer such as cough, hemoptysis, shortness of breath, sore mouth or tongue, dysphagia, tingling hands or feet, hair loss and pain. The response scale and scoring system are the same as for the EORTC QLQ-C30, and the administration's type and completion time-frame are similar (1-week). The use of diseasespecific questionnaires is not recommended without the 
administration of the QLQ-C30, since the content validity is based upon this combination. The completion of both questionnaires however, is estimated to take 11-12 minutes. The main scales are focusing on disease-related symptoms and treatment-toxicity. It has been validated in 1994 and validated in a group of 883 lung cancer patients. It showed good psychometric properties: the dyspnea score, for example, showed a Cronbach's alpha coefficient greater than 0.80 in many analysis (37). The module has been translated into more than 60 languages. A project updating this module to reflect aspects relevant in the newly available diagnostic and therapeutic options is underway and will be described in a separate section.

\section{Functional Assessment of Cancer Therapy-General Version (FACT-G) and Lung Cancer version (FACT-L)} The FACT-G is a 27 -item cancer-specific instrument divided in four domains: physical, social, emotional, and functional wellbeing, and a total score (38). All the items are rated on a 5-point Likert scale, and are not in the format of questions, but statements. The FACT-G was developed to measure QoL in cancer patients receiving therapy. The FACT family of instruments follows a core plus module design. The FACT-L is a 10-item lung cancer-specific module that supplements the FACT-G resulting a 37-item questionnaire, and includes 5 sub-scales self-assessment tool developed to measure lung cancer patients' QOL in the last 7 days, with each sub scale using a 0 to 4 Likert scale. FACT-G is scored by summing the individual scale scores, with higher scores indicating better QoL. There is also the option to calculate a Trial Outcome Index (TOI) which is the sum of the physical well-being (PWB), functional well-being (FWB), and any site-specific subscale (39). The FACT-L tool includes four generic cancer-specific domains and one lung cancer-specific subscale, and it has been reported to take 10 minutes to complete. These questionnaires are designed for patient self-administration, but can also be administered by interview format. The general subscales define PWB, social/family well-being, emotional well-being, and FWB. The fifth sub-scale describes the patient's pulmonary functionality including shortness of breath, loss of weight, and chest tightness $(40,41)$. Two points on the lung cancer subscale are considered clinically significant. A comparison with the EORTC QLQ-C30 has been recently published suggesting to use the decision tree presented (42). In lung cancer surgical patients, it has been less used compare to the extensive use in oncological setting (43-45).

\section{Lung Cancer Symptom Scale (LCSS)}

The LCSS (46) is a disease-specific measure of QoL, particularly used in clinical trials and contains both a patient and an observer section. The original LCSS was developed in 1985 at Memorial Sloan-Kettering Cancer Center. The patient form is a 9-item questionnaire which evaluates seven symptoms (appetite, fatigue, cough, shortness of breath, blood sputum, pain and general symptom burden), and two summary items that address functional activity and global QoL associated with lung cancer. This questionnaire, compare to the previous ones, doesn't address toxicity of treatment and only minimally refers to psychological issues. The LCSS observer's section assesses the same six symptoms excluding the general symptom burden. Conversely to the tools described before, it is referring to the past day and each item is scored on a $100-\mathrm{mm}$ VAS (where $0=$ worst, $100=$ best), where the score is the length of the line marked by the patient. It has also been validated with a Numerical Rating Scale (NRS), with an 11-pt response category format (47). It takes 4-8 minutes the first time to understand the VAS, but less for the following administration. The observer part takes 2 minutes to complete. As shorter and very sensitive to change, it is one of the most validated measure in oncological setting (48).

\section{The future of QOL tools}

\section{The EORTC QLQ-LC29 Project}

The recent advantages in the lung cancer treatment and the introduction of new radiotherapy techniques as targeted anti-cancer agents, made clear that the LC13 should be supplemented with new items to assess the effects and side-effects of the new various therapeutic options that are currently available to lung cancer patients $(49,50)$. This project has also been endorsed by the European Society of Thoracic Surgeons highlighting the importance of having surgery-specific questions in a QoL questionnaire. The preparatory phases resulted in a provisional 48 -item module. The aim of the international, cross-cultural, multicenter phase III study was to test this provisional module regarding relevance, acceptability, comprehensibility, and completeness. An important result of the phase III study was that 12 out of the 13 original LC13 items could be retained in the updated version. Phase I generated relevant QoL issues using a mix of sources including the involvement of 108 lung cancer patients. Phase II transformed issues into questionnaire items. In an international multicenter study 
(phase III), patients completed both the EORTC QLQ-C30 and the 48-item provisional lung cancer module generated in phases I and II. The phase III study enrolled 200 patients with histologically confirmed lung cancer from 12 centers and generate a new LC29 questionnaire which for the first time contains a 5-item subscale specifically designed to address issues of patients who had surgery for lung cancer (e.g., pain, restriction in performance). An international, cross-cultural, multicenter phase IV study has concluded the recruitment and results are being published to fully validate the psychometric properties of the LC29 (51). Table 1 provides a summary of the methods and the most important results of the development process of the QLQLC29. Table 2 depicts the items to assess symptoms related to thoracic surgery. This is the first validated scale to assess and is addresses patients who underwent thoracic surgery. Thus, the surgical subscale is optional and thus the QLQLC29 follows a 24-plus-5-item administration mode. In any event, the QLQ-LC29 is the instrument of choice when the goal is to assess the impact of multimodal treatment for lung cancer that may comprise radio-chemotherapy, targeted therapy, immunotherapy and thoracic surgery.

\section{The EORTC Item Library}

To face the continue evolution of cancer therapy, the EORTC QoL group has also created a tool with the primary aim of being used during the development of new instruments: the item library (52). It quickly became evident that a given module might not include problems and symptoms of novel treatments that were not available when the questionnaire was initially being developed. A static questionnaire might not always be sufficient to meet the demands of quickly evolving treatment modalities, the database began to shift into its new role as the Item Library, an integrated and dynamic tool to supplement existing measures with new relevant items. The Item Library is accessible to users from academia and industry after being granted of a specific permission through the website (https:// www.eortc.be/itemlibrary/).

\section{The PROMIS project}

The US-developed PROMIS ${ }^{\circledast}$ (Patient-Reported Outcomes Measurement Information System) is a set of personcentered measures that evaluates and monitors physical, mental, and social health. PROMIS was established in 2004 within the National Institutes of Health (NIH).
These surveys can be tailored to any patient population and are based on item response theory to adapt to the specific symptoms of a patient. PROMIS utilizes a larger database with validated items to put a specific, reproducible, and validated score. One of its main features is that these modules may be easily incorporated into a web-based platform which can be link to most of the electronic medical patient records across countries, facilitating also multicentre studies. Measures were developed for children and adults, and has been translated into more than 40 languages. For most of the PROMIS measures, the reference population was the 2000 General US Census. The use of PROMIS surveys has also been recognized by the Center for Medical Technology Policy as one of most important patient-reported tools for cancer clinical studies (53). Scoring method for PROMIS domains is standardized to population-based distributions of scores, producing a mean of 50 and a standard deviation of 10 , and provide a population-based standard for comparison of individual patient scores (54). In a Scopus review, has demonstrated that orthopedic surgeons are widely using PROMIS measures, demonstrating how accurately and reliably assess PROs in multidisciplinary surgical publications (55).

A recent published work has piloted the feasibility of integrating PROs into the institutional Society of Thoracic Surgery General Thoracic Surgery Database (STS-GTSD) with the use of the PROMIS platform. PROMIS tools allow for computer adaptive testing, efficiently achieving precise measurement of health status domains with few items.

\section{Mode of administration}

In other specialties some studies have investigated the influence of the mode of administration of QoL questionnaires (paper, telephone-based, interviews, electronic) without finding any important effect $(56,57)$. The introduction of technology has changed the completely this field due to widespread Internet use and the ability to monitor symptoms on-line and remotely. It has also demonstrated to have a positive effect on clinical outcomes $(58,59)$. Although the electronic administration has been introduced in our settings (60-62), we still need to face the limitations that the still most lung cancer population is facing, being older and not always computer-literate. However, an important example is coming from the esophageal surgery: a recent study has demonstrated that post-operative symptom monitoring after major surgery is feasible and acceptable (63). 
Table 1 Development of the QLQ-LC29: phase 1 to 4

\begin{tabular}{ll}
\hline Phase & \multicolumn{1}{c}{ Method } \\
\hline $\begin{array}{l}\text { Phase 1: Generation of } \\
\text { issues }\end{array}$ & $\begin{array}{l}\text { Literature review, review of questionnaires } \\
\text { in the field, interviews with patients and } \\
\text { health care providers }\end{array}$
\end{tabular}

$$
\text { health care providers }
$$

Phase 2: Item generation and construction of a provisional questionnaire

Phase 3: International study to assess comprehensibility, acceptance, and relevance
Using the EORTC item library, questionnaires items were formulated that were compatible with the standard response scale $(1=$ not at all, $2=$ a little, $3=$ quite a bit, $4=$ very much)

200 patients from nine regions (Cyprus, Germany, Israel, Italy, Norway, Spain, Poland, Taiwan, UK) filled in the QLQ-C30 and the provisional updated lung cancer module. They rated each item regarding comprehensibility, acceptance, and relevance

Item selection criteria were specified in advance which took into account patients' ratings as well as each item's distribution properties, such as mean, range, floor/ ceiling effects, prevalence of 3 and 4 responses, and missing responses

523 patients from 12 regions (Belgium, Cyprus, Germany, Greece, Israel, Italy, Jordan, Norway, Spain, Poland, Taiwan, UK) filled in the QLQ-C30 and the QLQ-LC29. 195 patients filled in the questionnaire at a second assessment point

The goal was to determine the scale structure, and to assess reliability, validity (known-group differences) and responsiveness to change
Results

QLQ-LC13 was used in 240 clinical trials, among them 109 RCTs. 92/109 (84\%) of the RCTs addressed QoL in the abstract. In none of the studies major problems with the use of the QLQLC13 were reported

In addition, 25 questionnaires relating to respiratory diseases were reviewed

The literature review and the review of existing questionnaires yielded a total of $110 \mathrm{QoL}$ issues to be considered in the context of lung cancer

108 patients and 103 health care providers from nine countries evaluated this list of issues regarding relevance and whether each of the issues should be included in the questionnaire

The analyses found 53 issues as relevant

After correcting for overlap/redundancy (also with the QLQ-C30), the provisional module contained 48 items

12 out of the 13 original QLQ-LC13 items were retained

The 48-item questionnaire was translated into the following (with English as the source language): German, Greek, Hebrew, Italian, Mandarin (Taiwan Chinese), Norwegian, Polish, and Spanish

29 of the 48 items met the pre-specified threshold criteria

12 out of the 13 original QLQ-LC13 items were retained, and newly added items addressed side-effects of targeted therapy, immunotherapy, surgery, as well as fear of progression

Confirmatory factor analyses is best compatible with a scale structure comprising five multi-item scales (coughing, shortness of breath, dyspnea, fear of progression, hair problems, surgeryrelated symptoms) and 15 single items

Internal consistencies of all multi-item scales ranged between 0.73 and 0.86 , and test-retest reliabilities ranged between 0.82 and 0.97 . Four of the 5 multi-item scales yielded known group differences when patients with lower vs. higher Karnofsky Performance Status were contrasted $(\mathrm{P}<0.01)$. Three of the 5 multi-item scales showed responsiveness to change over time $(\mathrm{P}<0.05)$, so did 9 out of 15 single symptoms

QLQ, Quality of Life Questionnaire; RCT, randomized controlled trial; EORTC, European Organization for Research and Treatment of Cancer. 
Table 2 QLQ-LC29 subscale to assess symptoms related to thoracic surgery

\begin{tabular}{|c|c|c|c|c|}
\hline $\begin{array}{l}\text { Please answer the following questions only if you had surgery for } \\
\text { lung cancer }\end{array}$ & Not at all & A little & Quite a bit & Very much \\
\hline 55. Have you had pain in the area of surgery? & 1 & 2 & 3 & 4 \\
\hline 56. Has the area of your wound been oversensitive? & 1 & 2 & 3 & 4 \\
\hline $\begin{array}{l}\text { 57. Have you been restricted in your performance due to the } \\
\text { extent of surgery? }\end{array}$ & 1 & 2 & 3 & 4 \\
\hline 59. Has your scar pain interfered with your daily activities? & 1 & 2 & 3 & 4 \\
\hline
\end{tabular}

(C) Copyright 2016 EORTC Quality of life Group. All rights reserved. Phase IV completed. This scale should be used in conjunction with the EORTC QLQ-C30 and with the entire QLQ-LC29. This is a copyrighted instrument. Please consult https://qol.eortc.org/questionnaires/ for user agreements. QLQ, Quality of Life Questionnaire; EORTC, European Organization for Research and Treatment of Cancer.

\section{Conclusions}

Advantages in surgical techniques and postoperative therapies have changed radically in the last decades the life expectancy of lung cancer survivors. The perioperative journey has also faced changes affecting directly the patient experience, so that PROs need to more adaptable to these changes than in the past. The development of more specific surgery-related questionnaires and dynamic measures may help the thoracic surgeons to implement future research and practical use of QoL outcomes.

\section{Acknowledgments}

Funding: None.

\section{Footnote}

Provenance and Peer Review: This article was commissioned by the Guest Editor (Peter J. Kneuertz) for the series "Patient reported Outcomes in Thoracic Surgery: A new Frontier" published in Fournal of Thoracic Disease. The article has undergone external peer review.

Conflicts of Interest: All authors have completed the ICMJE uniform disclosure form (available at http://dx.doi. org/10.21037/jtd.2019.12.131). The series "Patient reported Outcomes in Thoracic Surgery: A new Frontier" was commissioned by the editorial office without any funding or sponsorship. CP serves as an unpaid editorial board member of Journal of Thoracic Disease from Sep 2016 to Aug 2020. GV, MK and CP are members of the EORTC QoL group. The update of the lung cancer module and the
Phase 4 study on the QLQ-LC29 have been funded by the EORTC. GV: reports personal fees from Roche, personal fees from Eisai, personal fees from Novartis, grants from Pfizer, grants from Breast Cancer NOW, grants from Yorkshire Cancer Research, grants from EORTC, outside the submitted work.

Ethical Statement: The authors are accountable for all aspects of the work in ensuring that questions related to the accuracy or integrity of any part of the work are appropriately investigated and resolved. Informed patient's consent was obtained.

Open Access Statement: This is an Open Access article distributed in accordance with the Creative Commons Attribution-NonCommercial-NoDerivs 4.0 International License (CC BY-NC-ND 4.0), which permits the noncommercial replication and distribution of the article with the strict proviso that no changes or edits are made and the original work is properly cited (including links to both the formal publication through the relevant DOI and the license). See: https://creativecommons.org/licenses/by-nc-nd/4.0/.

\section{References}

1. Cornwell LD, Echeverria AE, Samuelian J, et al. Videoassisted thoracoscopic lobectomy is associated with greater recurrence-free survival than stereotactic body radiotherapy for clinical stage I lung cancer. J Thorac Cardiovasc Surg 2018;155:395-402.

2. Gazala S, Pelletier JS, Storie D, et al. A systematic review and meta-analysis to assess patient-reported outcomes 
after lung cancer surgery. ScientificWorldJournal 2013;2013:789625.

3. Pompili C, Novoa N, Balduyck B. Clinical evaluation of quality of life: a survey among members of European Society of Thoracic Surgeons (ESTS). Interact Cardiovasc Thorac Surg 2015;21:415-9.

4. The World Health Organization Quality of Life assessment (WHOQOL): position paper from the World Health Organization. Soc Sci Med 1995;41:1403-9.

5. Bottomley A, Reijneveld JC, Koller M, et al. Current state of quality of life and patient-reported outcomes research. Eur J Cancer 2019;121:55-63.

6. Calvert M, Kyte D, Mercieca-Bebber R, et al. Guidelines for Inclusion of Patient-Reported Outcomes in Clinical Trial Protocols: The SPIRIT-PRO Extension. JAMA 2018;319:483-94.

7. Pe M, Dorme L, Coens C, et al. Statistical analysis of patient-reported outcome data in randomised controlled trials of locally advanced and metastatic breast cancer: a systematic review. Lancet Oncol 2018;19:e459-69.

8. U.S. Department of Health and Human Services FDA Center for Drug Evaluation and Research; U.S. Department of Health and Human Services FDA Center for Biologics Evaluation and Research; U.S. Department of Health and Human Services FDA Center for Devices and Radiological Health. Guidance for industry: patientreported outcome measures: use in medical product development to support labeling claims: draft guidance. Health Qual Life Outcomes 2006;4:79.

9. Bottomley A, Pe M, Sloan J, et al. Analysing data from patient-reported outcome and quality of life endpoints for cancer clinical trials: a start in setting international standards. Lancet Oncol 2016;17:e510-4.

10. Sprangers MA, Cull A, Bjordal K, et al. The European Organization for Research and Treatment of Cancer. Approach to quality of life assessment: guidelines for developing questionnaire modules. EORTC Study Group on Quality of Life. Qual Life Res 1993;2:287-95.

11. Juniper EF. Validated questionnaires should not be modified. Eur Respir J 2009;34:1015-7.

12. Pompili C, Brunelli A, Xiume F, et al. Prospective external convergence evaluation of two different quality-of-life instruments in lung resection patients. Eur J Cardiothorac Surg 2011;40:99-105.

13. Kuenstner S, Langelotz C, Budach V, et al. The comparability of quality of life scores. a multitrait multimethod analysis of the EORTC QLQ-C30, SF-36 and FLIC questionnaires. Eur J Cancer 2002;38:339-48.
14. Apolone G, Filiberti A, Cifani S, et al. Evaluation of the EORTC QLQ-C30 questionnaire: a comparison with SF-36 Health Survey in a cohort of Italian long-survival cancer patients. Ann Oncol 1998;9:549-57.

15. Ware JE, Jr., Sherbourne CD. The MOS 36-item shortform health survey (SF-36). I. Conceptual framework and item selection. Med Care 1992;30:473-83.

16. Pompili C, Brunelli A. Quality of life after lung resection is not associated with functional objective measures. Eur Respir J 2013;42:283-5.

17. Brunelli A, Socci L, Refai M, et al. Quality of Life Before and After Major Lung Resection for Lung Cancer: A Prospective Follow-Up Analysis. Ann Thorac Surg 2007;84:410-6.

18. Handy JJR, Asaph JW, Douville EC, et al. Does videoassisted thoracoscopic lobectomy for lung cancer provide improved functional outcomes compared with open lobectomy? Eur J Cardiothorac Surg 2010;37:451-5.

19. Moller A, Sartipy U. Associations between changes in quality of life and survival after lung cancer surgery. J Thorac Oncol 2012;7:183-7.

20. Rizk NP, Ghanie A, Hsu M, et al. A Prospective Trial Comparing Pain and Quality of Life Measures After Anatomic Lung Resection Using Thoracoscopy or Thoracotomy. Ann Thorac Surg 2014;98:1160-6.

21. EuroQol Group. EuroQol--a new facility for the measurement of health-related quality of life. Health Policy 1990;16:199-208.

22. EuroQol Research Foundation. EQ-5D-5L User Guide, 2019. Available online: https://euroqol.org/publications/ user-guides

23. Devlin NJ, Brooks R. EQ-5D and the EuroQol Group: Past, Present and Future. Appl Health Econ Health Policy 2017;15:127-37.

24. Johnson JA, Pickard AS. Comparison of the EQ-5D and SF-12 health surveys in a general population survey in Alberta, Canada. Med Care 2000;38:115-21.

25. Koide R, Kikuchi A, Miyajima M, et al. Quality assessment using EQ-5D-5L after lung surgery for non-small cell lung cancer (NSCLC) patients. Gen Thorac Cardiovasc Surg 2019;67:1056-61.

26. Bejjani J, Fiore JF Jr, Lee L, et al. Validity of the EuroQol-5 dimensions as a measure of recovery after pulmonary resection. J Surg Res 2015;194:281-8.

27. Aaronson NK, Ahmedzai S, Bergman B, et al. The European Organization for Research and Treatment of Cancer QLQ-C30: a quality-of-life instrument for use in international clinical trials in oncology. J Natl Cancer Inst 
$1993 ; 85: 365-76$.

28. Osoba D, Zee B, Pater J, et al. Psychometric properties and responsiveness of the EORTC quality of Life Questionnaire (QLQ-C30) in patients with breast, ovarian and lung cancer. Qual Life Res 1994;3:353-64.

29. Nicklasson M, Bergman B. Validity, reliability and clinical relevance of EORTC QLQ-C30 and LC13 in patients with chest malignancies in a palliative setting. Qual Life Res 2007;16:1019-28.

30. Pompili C, Velikova G, White J, et al. Poor preoperative patient-reported quality of life is associated with complications following pulmonary lobectomy for lung cancert. Eur J Cardiothorac Surg 2017;51:526-31.

31. Balduyck B, Hendriks J, Lauwers P, et al. Quality of life evolution after lung cancer surgery: A prospective study in 100 patients. Lung Cancer 2007;56:423-31.

32. Schulte T, Schniewind B, Walter J, et al. Age-related impairment of quality of life after lung resection for nonsmall cell lung cancer. Lung Cancer 2010;68:115-20.

33. Burfeind WR Jr, Tong BC, O'Branski E, et al. Quality of life outcomes are equivalent after lobectomy in the elderly. J Thorac Cardiovasc Surg 2008;136:597-604.

34. Bendixen M, Jørgensen OD, Kronborg C, et al. Postoperative pain and quality of life after lobectomy via video-assisted thoracoscopic surgery or anterolateral thoracotomy for early stage lung cancer: a randomised controlled trial. Lancet Oncol 2016;17:836-44.

35. Pompili C, Koller M, Velikova G, et al. EORTC QLQ-C30 summary score reliably detects changes in QoL three months after anatomic lung resection for Non-Small Cell Lung Cancer (NSCLC). Lung Cancer 2018;123:149-54.

36. Giesinger JM, Kieffer JM, Fayers PM, et al. Replication and validation of higher order models demonstrated that a summary score for the EORTC QLQ-C30 is robust. J Clin Epidemiol 2016;69:79-88.

37. Bergman B, Aaronson NK, Ahmedzai S, et al. The EORTC QLQ-LC13: a modular supplement to the EORTC Core Quality of Life Questionnaire (QLQ-C30) for use in lung cancer clinical trials. EORTC Study Group on Quality of Life. Eur J Cancer 1994;30A:635-42.

38. Cella DF, Tulsky DS, Gray G, et al. The Functional Assessment of Cancer Therapy scale: development and validation of the general measure. J Clin Oncol 1993;11:570-9.

39. Webster K, Cella D, Yost K. The Functional Assessment of Chronic Illness Therapy (FACIT) Measurement System: properties, applications, and interpretation. Health Qual Life Outcomes 2003;1:79.
40. Cella DF, Bonomi AE, Lloyd SR, et al. Reliability and validity of the Functional Assessment of Cancer TherapyLung (FACT-L) quality of life instrument. Lung Cancer 1995;12:199-220.

41. Cella D, Eton DT, Fairclough DL, et al. What is a clinically meaningful change on the Functional Assessment of Cancer Therapy-Lung (FACT-L) Questionnaire? Results from Eastern Cooperative Oncology Group (ECOG) Study 5592. J Clin Epidemiol 2002;55:285-95.

42. Luckett T, King MT, Butow PN, et al. Choosing between the EORTC QLQ-C30 and FACT-G for measuring health-related quality of life in cancer clinical research: issues, evidence and recommendations. Ann Oncol 2011;22:2179-90.

43. Ring AE, Cheong KA, Watkins CL, et al. A Randomized Study of Electronic Diary versus Paper and Pencil Collection of Patient-Reported Outcomes in Patients with Non-Small Cell Lung Cancer. Patient 2008;1:105-13.

44. Porter LS, Keefe FJ, Garst J, et al. Self-efficacy for managing pain, symptoms, and function in patients with lung cancer and their informal caregivers: associations with symptoms and distress. Pain 2008;137:306-15.

45. Videtic GMM, Reddy CA, Sorenson L. A prospective study of quality of life including fatigue and pulmonary function after stereotactic body radiotherapy for medically inoperable early-stage lung cancer. Support Care Cancer 2013;21:211-8.

46. Hollen PJ, Gralla RJ, Kris MG, et al. Quality of life assessment in individuals with lung cancer: testing the Lung Cancer Symptom Scale (LCSS). Eur J Cancer 1993;29A Suppl 1:S51-8.

47. Hollen PJ, Gralla RJ, Kris MG, et al. A comparison of visual analogue and numerical rating scale formats for the Lung Cancer Symptom Scale (LCSS): does format affect patient ratings of symptoms and quality of life? Qual Life Res 2005;14:837-47.

48. Ferrero C, Badellino S, Filippi AR, et al. Pulmonary function and quality of life after VMAT-based stereotactic ablative radiotherapy for early stage inoperable NSCLC: a prospective study. Lung Cancer 2015;89:350-6.

49. Koller M, Warncke S, Hjermstad MJ, et al. Use of the lung cancer-specific Quality of Life Questionnaire EORTC QLQ-LC13 in clinical trials: A systematic review of the literature 20 years after its development. Cancer 2015;121:4300-23.

50. Koller M, Hjermstad MJ, Tomaszewski KA, et al. An international study to revise the EORTC questionnaire for assessing quality of life in lung cancer patients. Ann Oncol 
2017;28:2874-81.

51. 26th Annual Conference of the International Society for Quality of Life Research. Qual Life Res 2019;28:1-190.

52. Kuli D BA, Whittaker C, van de Poll-Franse LV, et al. The Use of the EORTC Item Library to Supplement EORTC Quality of Life Instruments. The International Society for Pharmacoeconomics and Outcomes Research (ISPOR) 20th Annual European Congress; 2017 Nov 4-8; Glasgow, Scotland. 2017.

53. Basch E, Abernethy AP, Mullins CD, et al. Recommendations for incorporating patient-reported outcomes into clinical comparative effectiveness research in adult oncology. J Clin Oncol 2012;30:4249-55.

54. Garcia SF, Cella D, Clauser SB, et al. Standardizing patientreported outcomes assessment in cancer clinical trials: a patient-reported outcomes measurement information system initiative. J Clin Oncol 2007;25:5106-12.

55. Jones RS, Stukenborg GJ. Patient-Reported Outcomes Measurement Information System (PROMIS) Use in Surgical Care: A Scoping Study. J Am Coll Surg 2017;224:245-254.e1.

56. Weinberger M, Oddone EZ, Samsa GP, et al. Are healthrelated quality-of-life measures affected by the mode of administration? J Clin Epidemiol 1996;49:135-40.

57. Gundy CM, Aaronson NK. Effects of mode of administration (MOA) on the measurement properties of the EORTC QLQ-C30: a randomized study. Health Qual
Life Outcomes 2010;8:35.

58. Velikova G, Brown JM, Smith AB, et al. Computer-based quality of life questionnaires may contribute to doctorpatient interactions in oncology. Br J Cancer 2002;86:51-9.

59. Basch E, Deal AM, Kris MG, et al. Symptom Monitoring With Patient-Reported Outcomes During Routine Cancer Treatment: A Randomized Controlled Trial. J Clin Oncol 2016;34:557-65.

60. Pompili C, Franks KN, Brunelli A, et al. Patient reported outcomes following video assisted thoracoscopic (VATS) resection or stereotactic ablative body radiotherapy (SABR) for treatment of non-small cell lung cancer: protocol for an observational pilot study (LiLAC). J Thorac Dis 2017;9:2703-13.

61. Khullar OV, Rajaei MH, Force SD, et al. Pilot Study to Integrate Patient Reported Outcomes After Lung Cancer Operations Into The Society of Thoracic Surgeons Database. Ann Thorac Surg 2017;104:245-53.

62. Denis F, Basch E, Septans AL, et al. Two-Year Survival Comparing Web-Based Symptom Monitoring vs Routine Surveillance Following Treatment for Lung Cancer. JAMA 2019;321:306-7.

63. Avery KNL, Richards HS, Portal A, et al. Developing a real-time electronic symptom monitoring system for patients after discharge following cancer-related surgery. BMC Cancer 2019;19:463.

Cite this article as: Pompili C, Koller M, Velikova G. Choosing the right survey: the lung cancer surgery. J Thorac Dis 2020;12(11):6892-6901. doi: 10.21037/jtd.2019.12.131 